\title{
6.4-dB Enhancement of the Gain of a Raman-assisted Fiber Optical Parametric Amplifier Over the Sum of the Gains of Individual Amplifiers
}

\author{
S. H. Wang, ${ }^{1}$ Lixin Xu, ${ }^{2}$ P. K. A. Wai, ${ }^{1}$ and H. Y. Tam $^{3}$ \\ ${ }^{1}$ Photonics Research Centre and Department of Electronic and Information Engineering, \\ The Hong Kong Polytechnic University, Hung Hom, Hong Kong \\ Phone: +852 2766-6231,fax:+852 2362-8439, email: enwai@polyu.edu.hk \\ ${ }^{2}$ Department of Physics, University of Science and Technology of China, Hefei, 230026, China \\ ${ }^{3}$ Photonics Research Centre and Department of Electrical Engineering, The Hong Kong Polytechnic University, Hung Hom, Hong Kong
}

\begin{abstract}
We first reported a $6.4 \mathrm{~dB}$ enhancement of the gain of a hybrid Raman-assisted fiber optical parametric amplifier over the sum of the gains of the individual Raman and parametric amplifiers. (C)2008 Optical Society of America

OCIS codes: (060.2320) Fiber optics amplifiers and oscillators; (190.4380) Four-wave mixing; (190.5650) Raman effect.
\end{abstract}

\section{Introduction}

Hybrid optical amplifiers, such as erbium-doped fiber (EDF)/Raman hybrid amplifiers, are attractive in optical communications because of its abilities of tailoring gain profile, compensating fiber dispersion and loss, enhancing the optical signal-to-noise ratio [1]. The gain of a hybrid amplifier is typically less than the sum of the gains of its component amplifiers because of gain saturation effect. The difference between the gain of the hybrid amplifier and the sum of the gains of component amplifiers can be written as

$$
\Delta G=G_{\text {hybrid }}-\sum_{i=1}^{N} G_{i}
$$

where, $G_{\text {hybrid }}$ and $G_{i}, i=1, \ldots, \mathrm{N}$ are the peak overall gain of the hybrid amplifier and peak gain of the i-th component amplifier, respectively. A positive $\Delta G$ represents the additional gain enhancement while a negative $\Delta G$ represents a penalty when combining different kinds of amplifiers together. Recently, a hybrid amplifier known as Raman-assisted fiber optical parametric amplifiers (RA-FOPAs) have attracted much attention because of its flexibility in selecting parametric pump wavelengths and full utilization of the $\mathrm{C}+\mathrm{L}$ band in addition to the inherent advantages of FOPAs [2]. Besides amplifying the parametric pump, the signal and the idler, the distributed Raman gain also contributes a nonlinear phase shift to the phase-matching condition and thus can further enhance the parametric gain and operating range of the four-wave mixing (FWM) process [3]. Despite its promises, the best hybrid gain enhancement $\Delta G$ in RA-FOPA to date is $-2.0 \mathrm{~dB}$ [4].

In this paper, we demonstrated for the first time a gain enhancement of $6.4 \mathrm{~dB}$ when combining a Raman amplifier and a continuous-wave (CW) pumped FOPA over the sum of the gains of the individual amplifiers. The net gain and the maximum internal gain of the RA-FOPA are $21.6 \mathrm{~dB}$ and $33.6 \mathrm{~dB}$, respectively.

\section{Experiment}

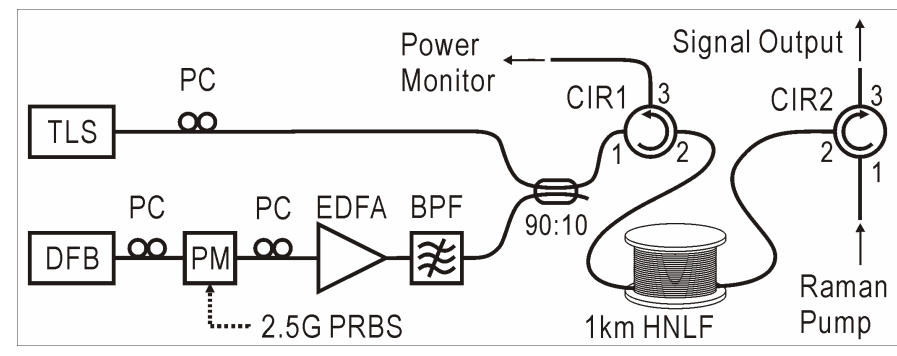

Fig. 1. Experimental setup of a Raman-assisted fiber optical parametric amplifier.

Figure 1 shows the experimental setup of the proposed RA-FOPA. A distributed feedback (DFB) laser diode served as the CW parametric pump. The parametric pump at wavelength $1554.9 \mathrm{~nm}$ was modulated by a phase modulator (PM) using pseudo-random binary sequence (PRBS) at $2.5 \mathrm{Gbps}$ in order to suppress stimulated Brillouin scattering (SBS). The phase-modulated parametric pump was then amplified by an ordinary EDFA. A 0.8 nm optical band-pass filter (BPF) was used to filter out the amplified spontaneous emission (ASE) noise. The 


\section{JThA13.pdf}

signal with an input power of $-14 \mathrm{dBm}$ was generated using a tunable laser source (TLS) and was combined with the parametric pump using a 90:10 pump-signal optical coupler. The combined signal was subsequently launched into a 1-km long commercial dispersion-flatten highly nonlinear fiber (HNLF) via an optical circulator (CIR1). The power of the parametric pump after CIR1 was measured to be $128 \mathrm{~mW}$ at $1554.9 \mathrm{~nm}$. The HNLF has a chromatic dispersion of $0.12 \mathrm{ps} / \mathrm{nm}^{2} \cdot \mathrm{km}$ at $1555 \mathrm{~nm}$, a dispersion slope of $0.01 \mathrm{ps} / \mathrm{nm}^{2} \cdot \mathrm{km}$, a nonlinear coefficient of 13.6 $\mathrm{W}^{-1} \cdot \mathrm{km}^{-1}$, and attenuation of $0.91 \mathrm{~dB} / \mathrm{km}$ at $1550 \mathrm{~nm}$. With $\mathrm{GeO}_{2}$ doped core, the HNLF has a Raman gain coefficient of $5.0 \mathrm{~W}^{-1} \cdot \mathrm{km}^{-1}$, which is 14 times larger than that of ordinary single mode fiber (SMF). Polarization controllers (PCs) were used to optimize the parametric process. A commercial 1455-nm CW fiber Raman laser serving as the Raman pump was launched into the HNLF in the counter-propagation direction via CIR2. The Raman pump power measured after CIR2 was $1.17 \mathrm{~W}$. The amplified signal and the idler were taken from the HNLF through CIR2. The pump on-off gains were measured in an optical spectrum analyzer (OSA) by comparing the signal with the pumps on and off. The total insertion loss for the input signal was measured to be $12.2 \mathrm{~dB}$, which is mainly due to the 90:10 coupler. The net wavelength-conversion efficiencies were measured in the OSA by comparing the signal input to the corresponding idler output.

\section{Results and Discussions}

Figure 2(a) shows the on-off gain of the Raman amplifier, parametric amplifier, and Raman-assisted parametric amplifier, respectively. Figure 2(b) shows the corresponding net wavelength conversion efficiency spectra with and without Raman assistance. In both figures, the parametric pump power after CIR1 and the Raman pump power after CIR2 were set to be $128 \mathrm{~mW}$ and $1.17 \mathrm{~W}$, respectively. The wavelength of parametric pump was selected as $1554.9 \mathrm{~nm}$ which corresponds to the wavelength of the Raman gain peak.
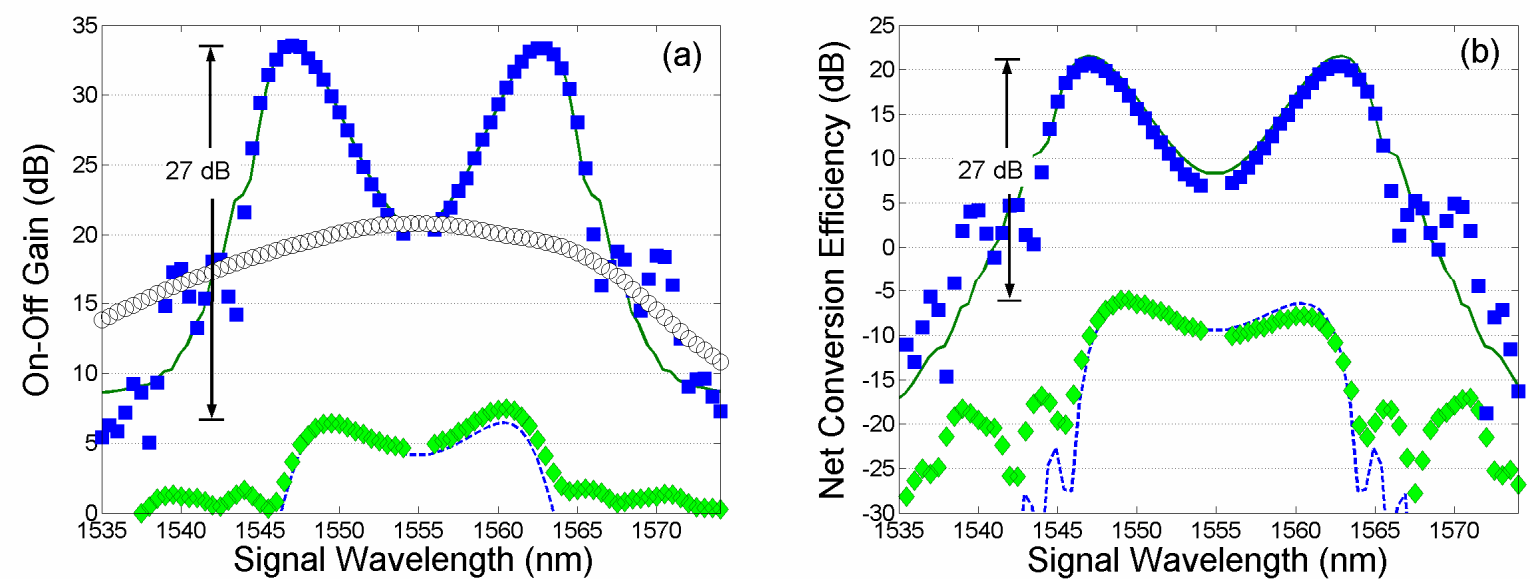

Fig. 2. (a) On-off gain and (b) wavelength conversion efficiency spectra for wavelength $1554.9 \mathrm{~nm}$. Squares, diamonds and circles represent the measured results of the Raman-assisted FOPA, FOPA without Raman pump, and Raman amplifier only, respectively. The Solid line and the dashed line were simulation results of the FOPA with and without Raman assistance, respectively. The parametric pump power after CIR1 was $128 \mathrm{~mW}$ and the Raman pump power after CIR2 was $1.17 \mathrm{~W}$.

From Fig. 2(a), the on-off gain of the parametric pump without Raman assistance has a peak value of $7.5 \mathrm{~dB}$ at $1560.5 \mathrm{~nm}$. The asymmetry in the parametric gain band is due to the contribution of an extra $1.1 \mathrm{~dB}$ Raman gain from the parametric pump to the gain band at longer wavelength. The Raman pump operating at $1455 \mathrm{~nm}$ can offer a maximum on-off gain of $20.8 \mathrm{~dB}$ at $1555 \mathrm{~nm}$ and the $3-\mathrm{dB}$ bandwidth is $23.5 \mathrm{~nm}$. When Raman amplification is combined with the parametric amplifier, the maximum gain of the hybrid amplifier is $33.6 \mathrm{~dB}$ and the 3-dB bandwidth for both gain bands is $\sim 4.5 \mathrm{~nm}$. The wavelengths of both gain peaks were shifted $2.6 \mathrm{~nm}$ away from the parametric pump wavelength of $1554.9 \mathrm{~nm}$ when compared with the FOPA without Raman assistance. Thus, there is $27.2 \mathrm{~dB}$ enhancement in the FOPA gain in the presence of the Raman pump. It is important to note that the hybrid amplifier provides an additional $6.4 \mathrm{~dB}$ gain enhancement when compare to the sum of individual peak gains of the Raman and parametric amplifiers. In Fig 2(b), the net conversion efficiency spectra had similar profiles to the hybrid on-off gain. The peak conversion efficiencies were $20.7 \mathrm{~dB}$ and $-6.0 \mathrm{~dB}$ with and without Raman assistance, respectively. Figures 2(a) and 2(b) also show the corresponding simulation results using the model described in [4]. We assumed that the Raman gain is uniform for all the wavelengths in the FWM process. In the simulations, we used the parameters given in Section 2, but we adjusted the Raman pump power to account for the depletion of the parametric pump power caused by the SBS effect. 


\section{JThA13.pdf}

\section{Raman-assisted Fiber Optical Parametric Amplifier Gain Contour Maps}

Figure 3(a) shows the measured peak gain values of the RA-FOPAs with different parametric pump powers and Raman pump powers. Second order least-square-fitting of the experimental data give the contour of the RA-FOPA gain. Figure 3(a) indicates that, for a given fiber, different choices of Raman and parametric pump powers can give the same overall gain. The circles show the results when the over-all gain of RA-FOPAs approximately equals the gain with only the Raman amplifier. For parametric pump below this limit, there is no significant change in the overall gain of the RA-FOPA. In Fig. 3(b), we shows the contour map of the gain difference between the peak gain of the hybrid amplifier and the sum of the peak gains of individual parametric and Raman amplifiers, which was obtained based on the data shown in Fig. 3(a).
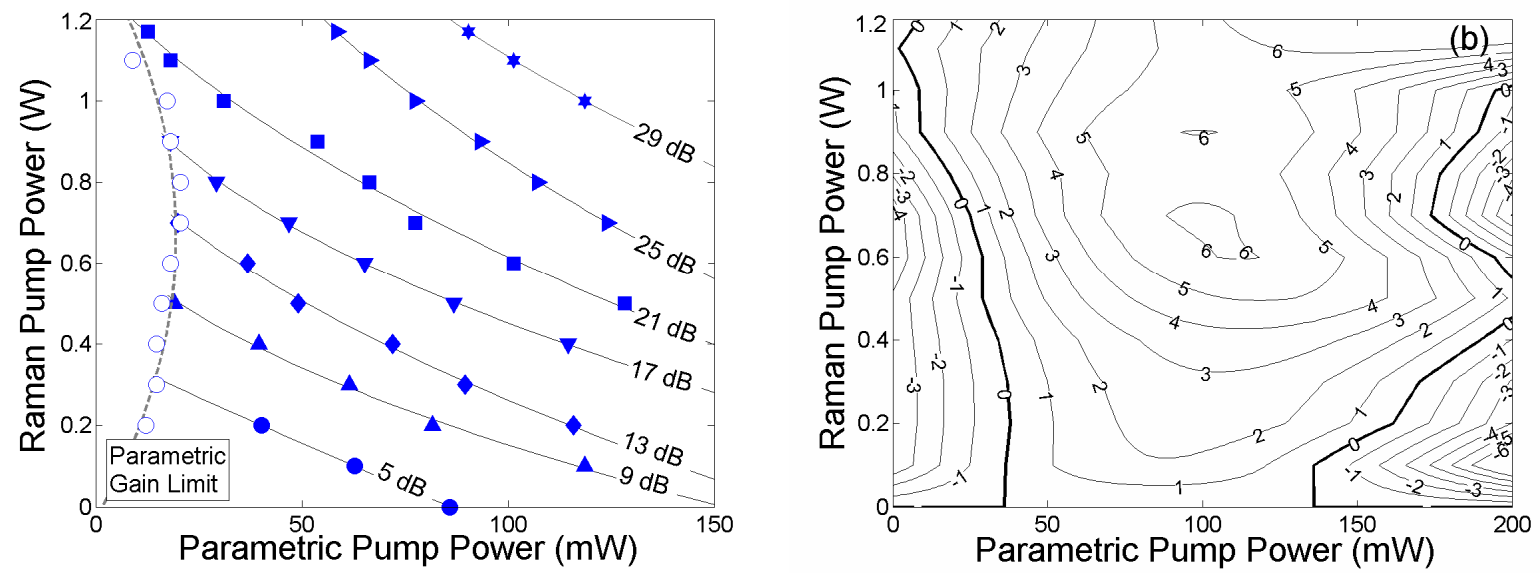

Fig. 3. The contour maps of (a) the RA-FOPA gains and (b) the hybrid gain differences. Solid symbols represent the overall gains under different combinations of Raman pump powers and parametric pump powers. Circles represent the results when the gain of RA-FOPAs equals to the gain with the Raman amplifier only.

As shown in Fig. 3(b), the maximum hybrid gain enhancement can be obtained at the center and upper-right portion of the contour map. For the same Raman pump power, the overall gain can be increased by increasing the parametric pump power. The hybrid gain difference $\Delta G$ will increase, then decrease and eventually becomes negative. This is due to the saturation of the Raman gain as the intensity of parametric pump becoming comparable to the Raman pump which is one of the main reasons of the negative hybrid gain differences observed in previous works $[2,4]$. From Fig. 3, a high hybrid gain enhancement can be obtained by carefully choosing the Raman and parametric pump powers to keep them out of the saturation region. For example, an $85 \mathrm{~mW}$ parametric pump can offer a $5 \mathrm{~dB}$ on-off gain without Raman assistance. If we add a $1.2 \mathrm{~W}$ Raman pump, we will get a 29-dB on-off gain with an improvement of $24 \mathrm{~dB}$. The gain enhancement of the hybrid amplifiers is a $6 \mathrm{~dB}$. In our experiments, the backward SBS caused parametric pump depletion and limited the parametric pump power. The SBS thresholds are related to the induced Raman pump powers in the Raman amplifiers [5]. In the experiment, we observed a SBS Stokes of $16.0 \mathrm{dBm}$ when the $2.5 \mathrm{G}$ phase modulated signal at $23.1 \mathrm{dBm}$ is amplified by a $1.0 \mathrm{~W}$ Raman pump.

\section{Conclusion}

In this paper we successfully demonstrated a $6.4 \mathrm{~dB}$ hybrid gain enhancement in a continuous-wave (CW) pumped Raman-assisted FOPA. The net gain and the maximum internal gain of the RA-FOPA are $21.6 \mathrm{~dB}$ and $33.6 \mathrm{~dB}$, respectively. We showed that large hybrid gain enhancement can be achieved in RA-FOPA by carefully choosing the Raman and parametric pump powers and keeping the parametric pump out of the saturation region and the parametric gain limit.

\section{References}

[1] H. Masuda et al., "Optical SNR enhance amplification in long-distance re-circulating loop WDM transmission experiment using $1580 \mathrm{~nm}$ band hybrid amplifier," IEEE Electron. Lett., 35, 411, (1999).

[2] D. A. Chestnut et al., "Raman-assisted fiber optical parametric amplifier and wavelength converter in highly nonlinear fiber," JOSA B, 19, 1901, (2002).

[3] S. H. Wang et al. "All-optical wavelength conversion using multi-pump Raman-assisted four-wave mixing," in OFC, OWQ1, (2007).

[4] J. F. L. Freitas et al., "Raman enhanced parametric amplifier based S-C band wavelength converter: Experiment and simulations," Opt. Comm., 255, 314, (2005).

[5] A. Koybyakov et al., "Stimulated Brillouin scattering in the Raman-pumped fibers: a theoretical approach,” IEEE JLT, 20, 1635, (2002). 